NASA Technical Memorandum 105915

AIAA-93-0482

\title{
An Improved Numerical Model for Wave Rotor Design and Analysis
}

Daniel E. Paxson

National Aeronautics and Space Administration

Lewis Research Center

Cleveland, Ohio

and

Jack Wilson

Sverdrup Technology, Inc.

Lewis Research Center Group

Brook Park, Ohio

Prepared for the

31st Aerospace Sciences Meeting

sponsored by the American Institute of Aeronautics and Astronautics

Reno, Nevada, January 11-14, 1993 


\title{
AN IMPROVED NUMERICAL MODEL FOR WAVE ROTOR DESIGN AND ANALYSIS
}

\author{
Daniel E. Paxson* \\ Lewis Research Center \\ Cleveland, Ohio \\ Jack Wilson"* \\ Sverdrup Technology, Inc. \\ Lewis Research Center Group \\ Cleveland, Ohio
}

\begin{abstract}
$\underline{\text { Abstract }}$
A numerical model has been developed which can predict both the unsteady flows within a wave rotor and the steady averaged flows in the ports. The model is based on the assumptions of one-dimensional, unsteady, perfect gas flow. Besides the dominant wave behavior, it is also capable of predicting the effects of finite tube opening time, leakage from the tube ends, and viscosity. The relative simplicity of the model makes it useful for design and optimization, as well as analysis, of wave rotor cycles for any application. This paper will discuss some details of the model and will then present comparisons between the model and two laboratory wave rotor experiments.
\end{abstract}

\subsection{Background}

The Wave Rotor is presently being investigated for use as a core gas generator for multi-spool gas turbine engines in order to achieve very high peak cycle temperatures and pressures. The device, shown schematically in Figure 1.1, uses unsteady waves to transfer energy directly to and from the working fluid through which the waves travel. It consists of a series of constant area passages (tubes) that rotate about an axis. Through rotation, the ends of the tubes are periodically exposed to various circumferentially arranged ports in which the flow is steady, but which initiate unsteady waves within the tubes. Because of the unsteady nature of the device, each tube of the wave rotor is periodically exposed to both hot and cold flow over roughly equal time periods. The mean temperature of the rotor material may therefore be expected to remain considerably below the peak cycle temperature. This characteristic, a comparatively low rotational speed for a given mass flow (as determined through analysis), and the absence of high torque loads, give the wave rotor potential advantages over the conventional gas generator.

Analyzing the wave rotor is difficult for several reasons. First, although the flows in the ports are steady, there is no steady state analysis which allows their properties to be

\footnotetext{
${ }^{t}$ Member AIAA

"Senior Member AlAA

Copyright 1992 ly the American Institute of Aeronautics anxl Astrouantics, Inc, No copyright is assetted in the United Stntes under Tille 17, U.S. Coxle. The U.S. Government has royalty-fiee license to exercise all rights under the copyright ctnimed herein for Governmental puposes. All other ights are reserved by the copytight owner.
}

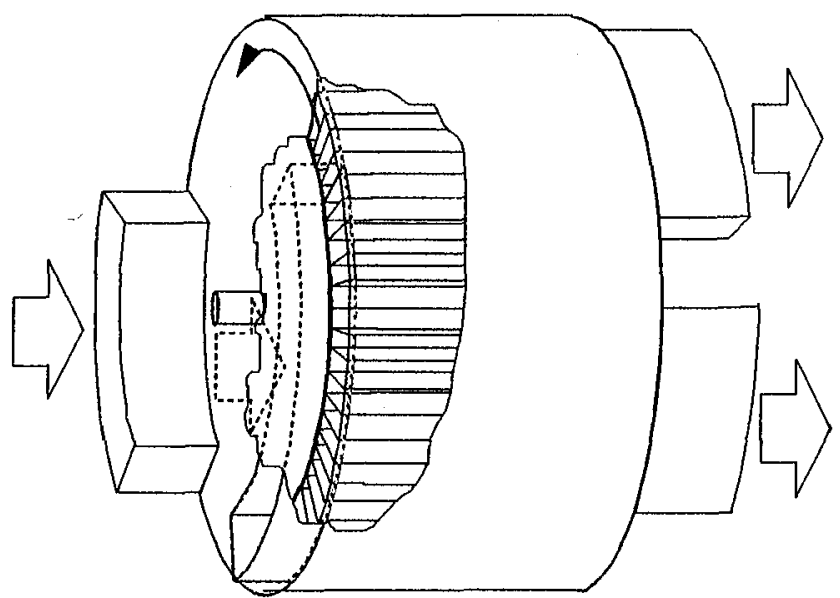

Figure 1.1 Wave Rotor Schematic

deternined. The wave rotor must therefore be analyzed by following the unsteady fluid dynamics that occur within one or several of the passages (tubes) as they rotate past the ports and walls. Second, the governing differential equations of motion in the tubes are hyperbolic and often have shock waves present. Closed form solutions of these equations do not generally exist, so numerical methods must be used. An effort is presently underway by the first author to develop such a numerical model or code. The initial phases of this effort were reported previously ${ }^{(1)}$. In that report the initial simplifying assumptions for the model were presented. For reference these were uniform cross sectional (i.e. one-dimensional), inviscid, perfect gas flow, with tubes which were instantaneously exposed to the ports in the manner of a ruptured shock tube diaphragm. Furthermore, it was assumed that since each tube in the wave rotor undergoes the same cycle, following one tube was equivalent to following them all. This amounts to an assumption that the tubes on the rotor do not interact. These simplifications reduced the modelling of the wave rotor to integrating the Euler Equations. A comparison was made between the model and an actual experimental wave rotor. It was found that although the model was qualitatively correct, there were significant discrepancies from the experiment.

The present paper is intended to highlight some of the modifications that have been made to the original model 
in order to predict the wave rotor behavior more accurately. In particular, the moclel, though still one dimensional, is now capable of including the effects of viscosity, leakage from the ends of the wave rotor tubes, and non-instantaneous opening of the tubes to the ports. These effects have been studied to some degree by other investigators. In particular, Eidelman ${ }^{(2)}$ studied finite opening time effects using a two dimensional inviscid code, and Taussig ${ }^{(3)}$ investigated all three effects with a one dimensional model. However, Eidelman's code is computationally expensive and thus not practical for analyzing entire wave rotor cycles. Furthermore, it does not account for leakage or viscosity. Taussig's code is not described in the literature with enough detail to implement. Furthermore, he assumes that the leakage effects are uncoupled from the wave phenomena, which from the present study, does not appear to be the case.

The paper will proceed by discussing the three modifications outlined above in some detail, then comparing the modified model to two simple wave rotor experiments.

\subsection{Finite Opening Time}

It is often assumed in wave rotor analysis that the ends of the tubes are instantaneously opened or closed as they rotate into or out of the various ports. This may be interpreted as assuming that the time required for the tube to pass from a fully open to a fully closed position (or vice-versa) is much less than the time required for a characteristic wave to travel down the length. In practice this is almost never the case, and the effects on the wave rotor performance due to this so called finite opening time can be substantial. Of course, the finite opening time effects are highly three dimensional; however, it is possible to obtain a reasonable estimate of them by modifying the boundary conditions for the one dimensional model. The discussion to follow will consider the left end of the wave rotor tube; however, it is understood that analogous conditions exist for the right end.

Consider Figure 2.1, where the left end of the tube is shown as it begins to enter the port region. Depending upon the port conditions, the flow will be either in or out. Looking first at the inflow scenario (a) it is imagined that the flow in the port itself is isentropic relative to some specified stagnation state, and that it instantaneously adjusts to conditions downstream (i.e. steady nozzle flow). Adjacent to this region is an imaginary "mixing region" where the fluid changes from a highly non-uniform distribution across the partially open tube on the left to some mixed out average on the right. The mixing is also imagined to occur instantaneously, and the extent of the mixing region is assumed negligible. Adjacent to this region is the first "cell" of the computing domain for which, at the present time, the conditions are completely known. Assuming that the flow is frictionless in the

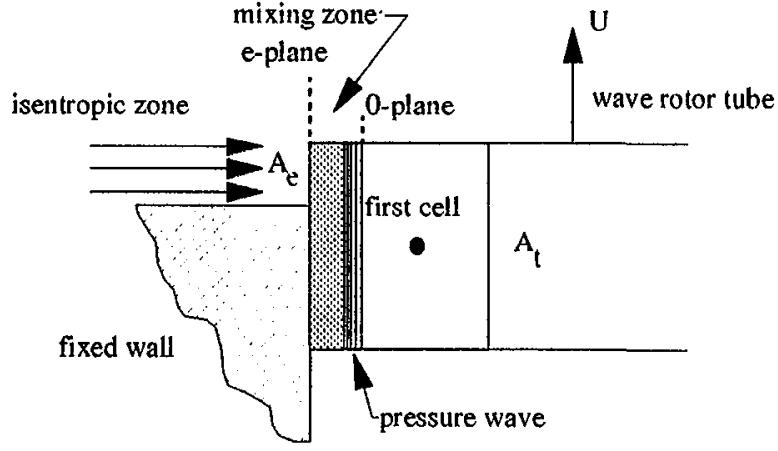

(a)

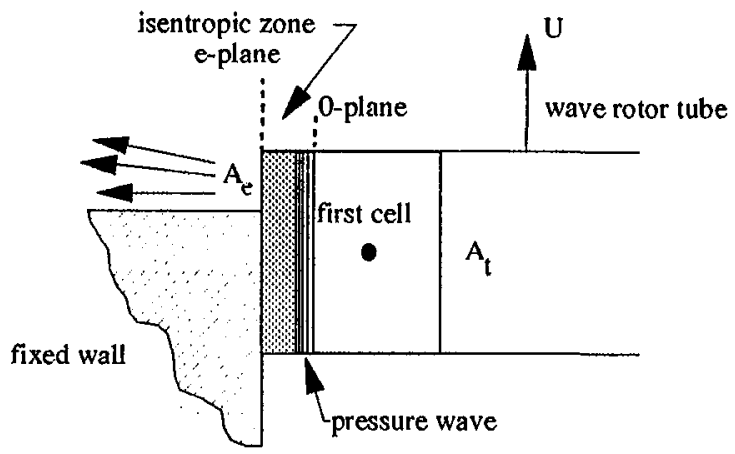

(b)

Figure 2.1 Finite Opening Time Model

mixing zone, and that the pressure is uniform across the left face, the following mass, momentum, and energy equations may be written for a perfect gas:

$$
\begin{aligned}
& \rho_{0} u_{0}=\rho_{e} u_{e} \frac{A_{e}}{A_{t}}=\phi_{m} \\
& p_{0}+\rho_{0} u_{o}^{2}=p_{e}+\rho_{e} u_{e}^{2} \frac{A_{e}}{A_{t}}=\phi_{u v o} \\
& \left(\frac{\gamma}{\gamma-1}\right) u_{0} p_{0}+\frac{1}{2} \rho_{0} u_{0}^{3}=\rho_{e} u_{e} H_{\frac{A_{e}}{A_{t}}}^{A_{t}}=\phi_{e},
\end{aligned}
$$

where $H$ is the total enthalpy. The subscripts 0 and $e$ in these equations designate the end and the beginning planes of the nixing zone respectively, as shown in Figure 2.1. If the pressure at the exit of the isentropic region, $p_{e}$ is specified, then given the known stagnation conditions of the duct and the area ratio of the exit to the tube, $\frac{A_{e}}{A_{1}}$, the conditions at the exit are completely known and the quantities $\phi_{\mathrm{s}}, \phi_{\mathrm{mon}}$, and $\phi_{\mathrm{e}}$ in equations $2.1-2.3$ can be calculated. Equations 2.1-2.3 may also be combined to yield the quadratic

$$
\left(\frac{\gamma+1}{2} \phi_{m}\right) u_{0}^{2}-\left(\gamma \phi_{m}\right) u_{0}+(\gamma-1) \phi_{e}=0
$$


This may be solved for $u_{4}$ (choosing the stibsonic value). With $u_{n}$ known, equations 2.1 and 2.2 may be used to obtain $p_{0}$ and $p_{0}$ so that the state at the right end of the mixing zone is completely known.

The conditions $p_{1}, p_{1}$, and $u_{1}$ in the first computational cell of the tube are also known. The difference between $p_{0}$ and $p$, will give rise to either a compression or expansion wave travelling to the right. The pressure ratio across the wave and the conditions in the first cell are sufficient information to analytically determine the velocity behind it, $u_{0}{ }^{*}$, using either the normal shock relations or isentropic centered expansion equations ${ }^{(4)}$. Since nothing has been said about the choice of $p_{e}$ it is not expected that $u_{0}{ }^{*}$ will be the same as $u_{0}$ calculated using equations 2.1-2.3. A function may be defined however as

$$
y\left(p_{e}\right)=u_{0}-u_{0}^{*}
$$

The proper choice of $p_{e}$ is that for which $y=0$. This cannot be found analytically but may be obtained using a convenient numerical root finding technique (e.g. the false point method(5) . The values of $p_{0}, p_{0}$, and $u_{0}$ obtained through the solution of equation 2.5 are then assigned to the left image cell of the computing space.

It is possible that the pressure $p_{e}$ drops to the point where the flow at the exit of the isentropic region is sonic but where equation 2.5 has not yet reached zero. In this case, it can no longer be assumed that $p_{\mathrm{e}}$ is uniform across the tube and equation 2.2 can no longer be used. Equations 2.1 and 2.3 remain valid however, and furthermore the values of $\phi_{\mathrm{m}}$ and $\phi_{\mathrm{w}}$, hecome frozen at their choked limits. As such, equation 2.3 may be rewritten as

$$
u_{0}=\frac{-\left(\frac{\gamma}{\gamma-1} p_{0}\right)+\sqrt{\left(\frac{\gamma}{\gamma-1} p_{0}\right)^{2}+2 \phi_{m} \phi_{e}}}{\phi_{m}}
$$

Thus, for any value of $p_{0}$ chosen, $u_{0}$ is known. Again, the conditions in the first cell are known so that another function

$$
y\left(p_{0}\right)=u_{0}-u_{0}^{*}
$$

may be defined and the value of $p_{0}$ for which $y=0$ may be found through the same method as equation 2.5 .

For outflow, consider Figure 2.1(b). Here, the duct is assumed to be a constant pressure region with the pressure, $p_{e}$ known. Adjacent to this is a fictitious zone in which the flow is isentropic and which, like the mixing zone above, adjusts instantaneously to flow conditions. Again, conditions in the first cell of the computing space are known. Assuming that the flow is unchoked at the exit of the isentropic zone, and subsonic in the first cell, steady isentropic flow laws require that between planes 0 and $e$, the Mach numbers are related by

$$
M a_{e}=-\left(\left(\frac{2}{\gamma-1}+M a_{0}^{2}\right)\left(\frac{p_{0}}{p_{e}}\right)^{\frac{\gamma-1}{\gamma}}-\frac{2}{\gamma-1}\right)^{\frac{1}{z}}
$$

Thus, if $p_{0}$ is specified then the exit Mach number is known. Since the state in the first cell is completely known, specification of $p_{0}$ also completely determines the state at the right plane of the isentropic zone (either through normal shock laws or laws for centered fans as in the inflow problem above). With $\mathrm{p}_{0}, \mathrm{u}_{0}$, and $\rho_{0}$ known, along with $p_{e}$ and the exit Mach number, $M_{e}, u_{e}$ and $p_{e}$ may be found using the relations for steady isentropic flow. Again, nothing has been said about the choice of $p_{i}$; however, in keeping with the assumptions laid down, the correct value is that for which mass continuity is maintained across the isentropic zone, namely

$$
y\left(p_{0}\right)=\rho_{c} u_{e} \frac{A_{e}}{A_{1}}-p_{0} u_{0}=0
$$

As with the inflow problem, equation 2.9 must be solved numerically using some iterative methol. The resulting values of the primitive variables for plane 0 are then assigned to the image cell of the computing space. If, in the process of solving equation 2.9, the exit Mach number drops below a value of -1.0 then the isentropic region is assumed choked and the values of $p_{\mathrm{e}}$ and $u_{\mathrm{e}}$ corresponding to this condition are used.

Additional possibilities must be considered for the outflow condition if the flow is negatively supersonic in the first cell. In this case $p_{0}$ is assigned a value, $p_{0}{ }^{*}$ which would create a stationary shock in plane $0^{(4)}$. The state at the exit is then found using equation 2.8 and the steady isentropic flow laws. The value of $y$ in equation 2.9 is then calculated. If this value is greater than zero then the iteration process may continue since it is now assured that there is some $p_{e}<p_{0}<p_{0}$ " for which $y=0$. If $y$ is less than zero the only remaining possibility is that the flow in the first cell is completely unaffected by the boundaries, In this case, the image cell will have no effect on the internal computations and it may simply be assigned the values of the first cell.

In the above discussion it is assumed known a priori whether the flow resulting from the imposition of boundary conditions will be inwaid or outward. In actuality, the code must anticipate the resulting flow and then apply the appropriate model. This may be done in a rather straightforward manner as follows. If the flow is assumed inward, then the minimum value of equation 2.5 is obtained by setting $p_{t}$ equal to the prescribed duct stagnation pressure. In this extreme case, $p_{e}$ is also the 
same as $p_{0}$ and the value of $y$ is not only minimum, but negative. The exit pressure, $p_{e}$ must be lowered from this value in order to bring equation 2.5 to zero. If, the value of $y$ is found to be positive, then the assumption of inflow was incorrect, and the outflow model must be used. It is noted here that in the code, the same prescribed boundary pressure is used whether the flow is in or out. Thus, for inflow, the pressure is interpreted as a stagnation value and an additional stagnation temperature is needed. For outflow, it is interpreted as a static pressure. An analogy may be made here if the wave rotor duct is viewed as a large reservoir or "tank". Flow into this tank simply loses its kinetic energy at the tank stagnation pressure (i.e. jet flow). Flow from the tank leaves isentropically at the stagnation pressure.

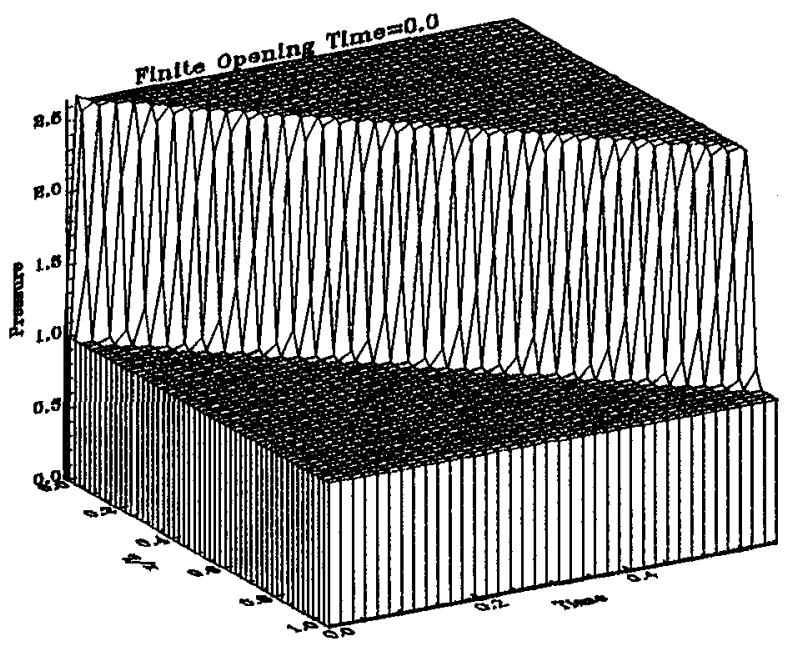

(a)

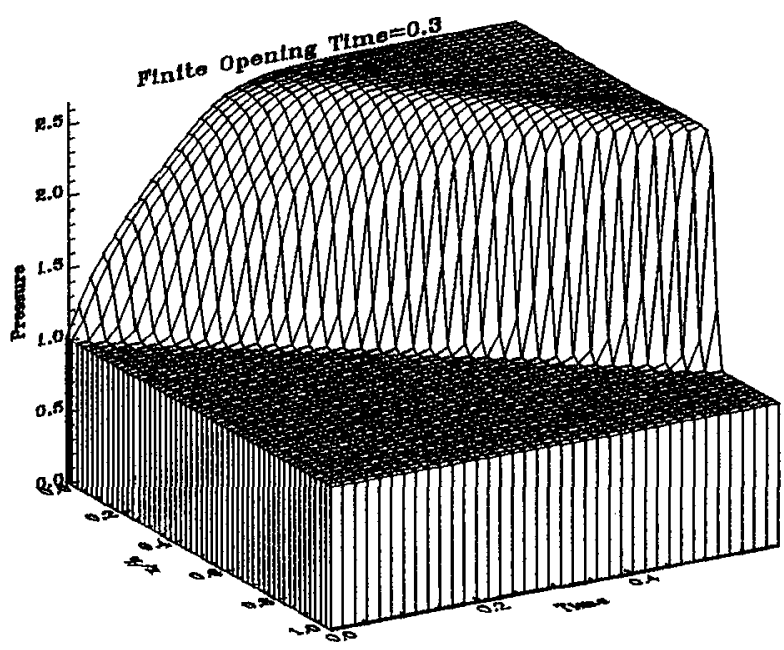

(b)

Figure 2.2 Finite Opening Time Effects

As an example of the application of finite opening time boundary conditions, Figure 2.2 shows a computed time dependent pressure distribution in a hypothetical wave rotor tube as the left side is exposed to a high pressure duct. The pressure has been normalized by the initial pressure in the tube and the time has been normalized by the quantity $\mathrm{L} / a$, where $\mathrm{L}$ is the tube length and $a$ is the initial speed of sound in the tube. For this calculation a cell spacing of $\Delta x / L=.02$ was used, with an associated time step of $\Delta t a / L=.008$. Figure 2(a) illustrates the scenario with instantaneous opening time. Here, a shock wave is formed immediately and travels rightward with a uniform speed $u_{\mathrm{sh}}=1.65$. Figure $2(\mathrm{~b})$ shows the same scenario with an opening time $t_{\text {npeu }} a / L=0.3$. Although it is not possible to claim quantitatively that the results are correct, it is fair to say that the applied boundary conditions appear to generate the anticipated area-averaged behavior in the tube. In particular, there is a certain amount of time required for an actual shock to form, and the speed at which it travels is slower than that in the case where the opening was instantaneous. It is this effect on wave speed that is critical for wave rotor design and analysis and, as will be shown later, the approach described above produces excellent agreement with experimental data.

\section{$\underline{3.0 \text { Source Terms }}$}

Aside from the effects of finite opening time, the fluid mechanics in the wave rotor tube are dominated by one dimensional, inviscid wave phenomena. As such, the Euler equations serve as a good starting point for predicting the time dependent behavior; however, there are several effects which, while secondary, are not negligible. In particular, it has been found that viscous effects, and the effects due to leakage of flow from the ends of the wave rotor tube to the casing can have a significant impact on the behavior of the flow. As with finite opening time, these effects are three dimensional; however, the one dimensional model can again be modified to account for them satisfactorily. The approach for both phenomena is to add source terms to the original homogeneous governing equations. The discussion to follow will first consider the addition of source terms in general to the Euler equations, and the requirements for high order accuracy. Following this, the particular form of the source terms used for modelling viscous effects (friction) and leakage effects will be presented.

The particular form of the modified Euler equations to be considered is one in which the source term, like the flux vector, is a function of the original conserved vector. That is,

$$
\frac{\partial \underline{w}}{\partial t}+\frac{\partial F(w)}{\partial x}=\underline{S}(\underline{w})
$$

For reference, the vectors $\underline{w}$ and $\underline{F}$ have the respective perfect gas forms: 


$$
\begin{aligned}
& \underline{w}=\left[\begin{array}{l}
\rho \\
\rho u \\
\rho\left(e+\frac{u^{2}}{2}\right)
\end{array}\right] \\
& \underline{F}=\left[\begin{array}{l}
\rho u \\
p+\rho u^{2} \\
\rho u\left(h+\frac{u^{2}}{2}\right)
\end{array}\right]=\left[\begin{array}{l}
w_{2} \\
(\gamma-1) w_{3}+\left(\frac{3-\gamma}{2}\right) \frac{w_{2}^{2}}{2 w_{1}} \\
\frac{w_{2}}{w_{1}}\left(\gamma w_{3}-\left(\frac{\gamma-1}{2}\right) \frac{w_{2}^{2}}{w_{1}}\right.
\end{array}\right]
\end{aligned}
$$

where $h$ and $e$ are the specific enthalpy and internal energy respectively, and the numbered subscripts refer to the elements of $\underline{w}$. The form of $\underline{S}(\underline{w})$ has yet to be specified.

The straightforward approach to numerically integrating these equations is to expand all of the derivatives about a point, i.e.

$$
\begin{aligned}
& \frac{\underline{w}_{-1}^{n+1}-\underline{w}_{1}^{n}}{\Delta t}=\frac{\partial \underline{w}_{i}^{n}}{\partial t}+\frac{\partial^{2} \underline{w}_{i}^{n}}{\partial t^{2}} \frac{\Delta t}{2}+O\left(\Delta t^{2}\right) \\
& \frac{F_{t+1}^{\prime \prime}-\underline{F}_{-1}^{n}}{2 \Delta x}=\frac{\partial F_{1}^{n}}{\partial x}+O\left(\Delta x^{2}\right)
\end{aligned}
$$

where the subscript $n$ indicates the discrete temporal index $n \Delta t$ and $i$ the spatial index $i \Delta x$. Adding equations 3.4 and 3.5 , then subtracting the source term $S_{1}$ from both sides of the resulting equality yields

$$
\begin{aligned}
\frac{\underline{w}_{i}^{n+1}-\underline{w}_{i}^{n}}{\Delta t}+\frac{\underline{F}_{i+1}^{n}-\underline{F}_{-1}^{n}}{2 \Delta x}-\underline{S}_{-1} & =\frac{\partial \underline{w}_{1}^{n}}{\partial t}+\frac{\partial \underline{F}_{-1}^{n}}{\partial x}-\underline{S}_{1} \\
& +\frac{\partial^{2} \underline{w}_{-1}^{n}}{\partial t} \frac{\Delta t}{\partial t^{2}}+O\left(\Delta t^{2}\right) .
\end{aligned}
$$

The numerical scheme on the left hand side approximates the actual differential equation on the right up to a first order truncation error of order $O(\Delta t)$. This can be improved by finding a suitable approximation for $\frac{\partial^{2} \underline{w}_{i}^{u}}{\partial t^{2}}$. After Lax and Wendroff( ${ }^{(5)}$, taking the time derivative of equation 3.1 at the point $n \Delta x$, ist yields

$$
\frac{\partial^{2} \underline{w}}{\partial t^{2}}+\frac{\partial^{2} \underline{F}}{\partial x \partial t}=\frac{\partial \underline{S}}{\partial t}
$$

where the subscripts have temporarily been dropped. Using the Jacobians defined as

$$
[\mathrm{A}]=\frac{\partial \underline{\mathrm{F}}}{\partial \underline{\mathrm{w}}} \quad[\mathrm{B}]=\frac{\partial \underline{\mathrm{S}}}{\partial \underline{\mathrm{w}}}
$$

equation 3.7 may be rewritten as

$$
\frac{\partial^{2} \underline{w}}{\partial t^{2}}+\frac{\partial}{\partial x}\left([A] \frac{\partial w}{\partial t}\right)=[B] \frac{\partial \underline{w}}{\partial t}
$$

or, upon substitution of equation 3.1

$$
\begin{aligned}
\frac{\partial^{2} \underline{w}}{\partial t^{2}}=\frac{\partial}{\partial x}\left([\mathrm{~A}] \frac{\partial \underline{\mathrm{F}}}{\partial \mathrm{x}}\right) & -\frac{\partial}{\partial \mathrm{x}}([\mathrm{A}] \underline{\mathrm{S}}) \\
& -[\mathrm{B}] \frac{\partial \vec{F}}{\partial \mathrm{x}}+[\mathrm{B}] \underline{\underline{S}} .
\end{aligned}
$$

Multiplying equation 3.10 by $\Delta t / 2$, replacing the spatial derivatives on the right with their numerical equivalents (note that these need only be first order accurate), and adding this to equation 3.6 yields

$$
\begin{aligned}
& \frac{\underline{w}_{i}^{n+1}-\underline{w}_{i}^{n}}{\Delta t}+\frac{\underline{F}_{i+1}^{n}-\underline{F}_{-1-1}^{n}}{2 \Delta x}-\underline{S}_{i}^{n} \\
& -\frac{\Delta t}{2}\left([\mathrm{~A}]_{i+1 / 2}^{\prime \prime} \frac{\frac{F}{i+1}^{\prime \prime}-F_{-1}^{\prime \prime}}{\Delta x^{2}}-[A]_{1-1 / 2} \frac{F_{i}^{\prime \prime}-F_{i-1}^{\prime \prime}}{\Delta x^{2}}\right) \\
& +\frac{\Delta t}{2}\left(\frac{[A]_{i+1}^{n} \underline{S}_{i+1}^{n}-[A]_{i-1}^{n} S_{i-1}^{n}}{2 \Delta x}\right)+\frac{\Delta t}{2}\left([B]_{i}^{n} \frac{F_{i+1}^{n}-F_{i-1}^{n}}{2 \Delta x}\right) \\
& -\frac{\Delta \mathrm{t}}{2}[\mathrm{~B}]_{\mathrm{i}}^{\mathrm{n}} \underline{\mathrm{S}}_{\mathrm{i}}^{\mathrm{n}}=\frac{\partial \underline{\mathrm{w}}_{\mathrm{i}}^{\mathrm{n}}}{\partial \mathrm{t}}+\frac{\partial \underline{\mathrm{F}}_{\mathrm{i}}^{\prime \prime}}{\partial \mathrm{x}}-\underline{\mathrm{S}}_{\mathrm{i}}^{\mathrm{n}}+\mathrm{O}\left(\Delta \mathrm{t}^{2}, \Delta \mathrm{x}^{2}\right)
\end{aligned}
$$

Here, the Jacobian $[A]_{i+1 / 2}$ is the mean of two adjacent cells, i.e. $1 / 2\left([A]_{1+1}+[A]_{1}\right)$. The left hand side of this equality, when set equal to zero is the second order accurate numerical approximation to equation 3.1. This may be rearranged and compacted to the form

$$
\underline{w}_{-1}^{n+1}=\underline{w}_{-1}^{n}-\left(f_{-1+12}^{n}-\underline{f}_{-1-1 / 2}^{n}\right) \frac{\Delta t}{\Delta x}+\underline{s}_{-1}^{n} \Delta t
$$

where

$$
f_{-1 / 2}^{\prime \prime}=\frac{F_{i+1}^{n}+F_{-1}^{n}}{2}-\frac{\Delta t}{\Delta x}[A]_{i+1 / 2}^{n} \frac{F_{-1+1}^{n}-F_{-1}^{n}}{2}
$$

and

$$
\begin{aligned}
\underline{S}_{-i}^{n}=\underline{S}_{i}^{n} & -\frac{\Delta t}{4 \Delta x}\left([A]_{i+1}^{n} \underline{S}_{-1+1}^{n}-[A]_{i-1}^{n} \underline{S}_{-1}^{n}\right) \\
& -\frac{\Delta t}{4 \Delta x}[B]_{i}^{n}\left(\underline{F}_{-1+1}^{n}-F_{-1-1}^{n}\right)+\frac{\Delta t}{2}[B]_{i-1}^{n} \underline{S}_{-1}^{n}
\end{aligned}
$$

The numerical flux estimate for the cell face, $f_{j+1 / 2}$ in equation 3.13 , has been chosen in this particular fashion 
because it is identical to the conservative form found when numerically approximating the homogeneous version of equation 3.1. It is found in practice however, that this estimation produces physically unrealistic oscillations in the solution. To overcome this problem, equation 3.13 is replaced with a high resolution, upwinding flux estimate guaranteed to produce monotonic results in the homogeneous Euler equations. This is based upon Roe' $\mathrm{s}^{(7)}$ approximate Riemann solver and is described in detail in a previous report ${ }^{(1)}$. The numerical source estimate, $s$ in equation 3.14 is seen to be based entirely on central differences. This is physically intuitive since the sources should not have any directional bias. There is no way to formally guarantee that the resulting scheme is monotonic since equation 3.1 is not conservative (see Lax [3]); however, the approach taken above will at least approach monotonicity as the strength of the source term is brought to zero. Furthermore, it is found in practice that this method retains very sharp resolution of discontinuities. This is illustrated in Figure 3.1 where the method has been applied to the scalar equation

$$
\frac{\partial u}{\partial t}+u \frac{\partial u}{\partial x}=c u|u|
$$

The figure shows the initial profile, a half sine wave, corresponding to $t=0$. Also shown in the figure are the profiles at $\mathrm{t}=0.5$ for three different values of the constant $c$ in equation 3.15 , corresponding to positive, negative and zero source term strengths. For this computation spatial and temporal spacings of $\Delta x=0.01$ and $\Delta t=0.005$ respectively were used. There is no analytical solution by which to verify the results; however, it is seen that the shock remains sharp, and the pulse height and speed vary as expected. Furthermore, although not shown here, the stability of the scheme appears unaffected since the maximum allowable CFL number was found to be unchanged even for large values of $c$.

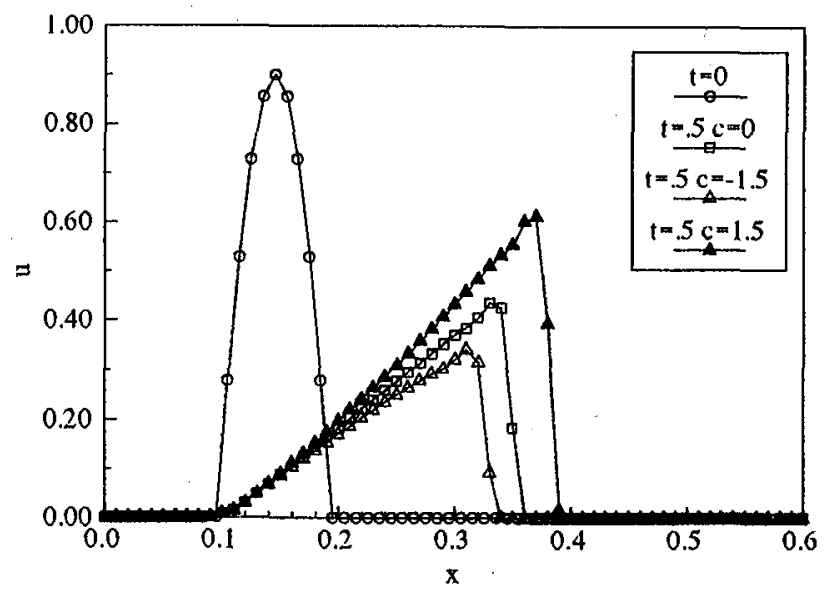

Figure 3.1 Burger's Equation With a Source

\section{$\underline{3.1 \text { Viscous Source Terms }}$}

The major effect of viscosity in the wave rotor tube is that it creates boundary layers near the walls. These in turn alter the behavior of the core flow which remains inviscid. Naturally, a one dimensional model cannot analyze this phenomenon per se; however, if the terms in the one dimensional equations of motion are viewed as inlegral averages of the values over the tube cross section, then the viscous effects (except in the vicinity of shock waves) become confined to the shear stress at the wall, and the momentum equation takes the form

$$
\frac{\partial}{\partial t} p u+\frac{\partial}{\partial x}\left(p+\rho u^{2}\right)=-4 \frac{\tau_{\text {wau }}}{D_{h}}
$$

Here, $D_{h}$ is the hydraulic diameter defined as $\frac{4 A}{W_{p}}$ where $A$ is the cross sectional area of the tube and $W_{p}^{n}$ is the wetted perimeter. The task now becomes one of obtaining a reasonable estimate of $\tau_{\text {wnll. }}$. Some researchers [4] have used correlations corresponding to fully developed turbulent pipe flow; however, considering typical tube dimensions and the amount of time that the gas in the tube is actually moving, this does not seem to be an appropriate approach. An alternate estimate of the wall shear stress may obtained by considering each discreet computational region of the tube to be a suddenly decelerated flat plate (or inversely a stationary plate with suddenly accelerated fluid). The solution to such a problem, for incompressible flow, is well known ${ }^{(8)}$ and has the form

$$
\begin{gathered}
\overrightarrow{\mathrm{u}}=U_{\infty} \operatorname{erf}(\eta) \\
\eta=\frac{y}{2 \sqrt{v \mathrm{t}}} .
\end{gathered}
$$

Here, $\mathrm{U}_{\infty}$ is the speed of the freestream flow, $v$ is the kinematic viscosity, $y$ is the distance from the wall, and erf is the error function. For the problem at hand, the freestream velocity is replaced by the local velocity in the tube. The wall shear stress has the form

$$
\left.\tau_{\text {wall }}=\mu_{\frac{\partial u}{\partial y}}\right)_{y=0}=\frac{2 u \mu}{\sqrt{v t \pi}} .
$$

A characteristic time associated with the wave rotor is that required for a wave to travel down the tube, namely $t=L / a^{*}$ where $L$ is the tube length and $a^{\prime \prime}$ is the speed of sound at some characteristic stagnation state. Substituting this into equation 3.1.3, assuming constant viscosity, and nondimensionalizing by $p^{*}, p^{*}$, and $a^{*}$, the momentum equation may now be written as 


$$
\begin{aligned}
& \frac{\partial}{\partial \mathbf{t}^{\prime}}\left(\rho^{\prime} \mathbf{u}^{\prime}\right)+\frac{\partial}{\partial \mathrm{x}^{\prime}}\left(\frac{p^{\prime}}{\gamma}+\rho^{\prime} \mathbf{u}^{\prime 2}\right)= \\
& -\alpha \frac{8}{\sqrt{\pi}} \frac{\sqrt{\frac{v_{1} L}{a_{1}}} u^{\prime} \sqrt{\rho^{\prime}}}{D_{h}}
\end{aligned}
$$

Since the above analysis is only an estimate of the wall shear behavior, the term $\alpha$ appearing in equation 3.1 .4 has been added as a constant to be determined through

comparison with experimental data. The ratio $\frac{\sqrt{\frac{v_{1} L}{a_{1}}}}{D_{h}}$ is a measure of the penetration depth of the boundary layer into the tube and is generally small. When multiplied by the additional constant terms however, the contribution to the right hand side of equation 3.1 .4 becomes significant (on the order of 0.1 ).

\subsection{Leakage Source Terms}

Leakage in the wave rotor occurs from the tube ends into the rotor casing and vice versa, as illustrated in Figure 3.2.1. As such, the leakage source terms are applied only to the first and last cells in the computing domain and they affect only the continuity and energy equations. Consider the control volume shown in Figure 3.2.2. As with the boundary conditions, it is assumed that the leakage flow adjusts instantaneously to the conditions in the cell. The leakage gap is imagined as distributed uniformly along the cell in the manner of porosity. Assuming that the flow is out of the tube, the isentropic nozzle flow equation may be used to write

$$
\begin{aligned}
\mathrm{m}_{\text {leak }} & =\left(\frac{2 \gamma}{\gamma-1}\right)^{1 / 2} \frac{\mathrm{dA}_{1}}{\mathrm{dx}} \Delta \mathrm{x} \sqrt{\mathrm{p} \rho} \mathrm{f}\left(\frac{\mathrm{p}_{\text {cav }}}{\mathrm{p}}\right) \\
\mathrm{f}\left(\frac{\mathrm{p}_{\text {cav }}}{\mathrm{p}}\right) & =\left(\left(\frac{\mathrm{p}_{\text {cav }}}{\mathrm{p}}\right)^{2 / \gamma}-\left(\frac{\mathrm{p}_{\text {cav }}}{\mathrm{p}}\right)^{\frac{\gamma+1}{\gamma}}\right)^{1 / 2}
\end{aligned}
$$

where $\frac{d A_{1}}{d x}$ is the leakage area per unit length of the tube, and $p_{\text {cav }}$ is the pressure in the center cavity of the wave rotor. Incorporating this equation into the continuity and energy equations yields

$$
\frac{\partial w_{1}}{\partial t}+\frac{\partial F_{1}}{\partial x}=-C_{1} \sqrt{p \rho} f\left(\frac{p}{p}\right)
$$

$$
\frac{\partial w_{3}}{\partial t}+\frac{\partial F_{3}}{\partial x}=-C_{1} \sqrt{p \rho} H A\left(\frac{p_{\text {cav }}}{p}\right)
$$

where $C_{1}$ is the leakage coefficient $C_{1}=\left(\frac{2 \gamma}{\gamma-1}\right)^{1 / 2} \frac{d\left(\frac{A_{1}}{A}\right)}{d x}$, and $A$ is the cross sectional area of the tube. The value

of $\frac{d\left(\frac{A_{1}}{A}\right)}{d x}$ may be estimated by assuming a rectangular tube cross section in which case it takes on the value $\frac{2 \delta}{h \Delta x}$, where $\delta$ is the leakage gap, $h$ is the tube height, and $\Delta \mathrm{x}$ is the numerical cell size. If the flow becomes choked in the nozzle equation then the value of the flow function $f$ in equation 3.2.1 becomes frozen at the choked limit. If the cavity pressure is higher than the cell pressure, then the pressures and densities in equation 3.2.1 are replaced with the cavity values and the cavity pressure in the flow function is replaced with the cell pressure.

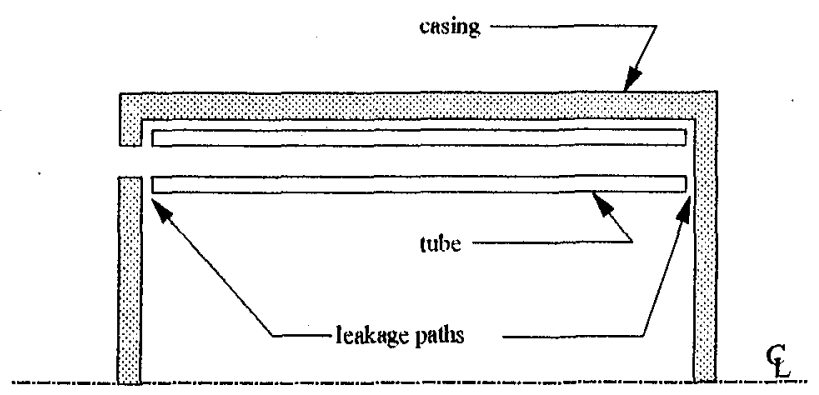

Figure 3.2.1 Leakage Paths

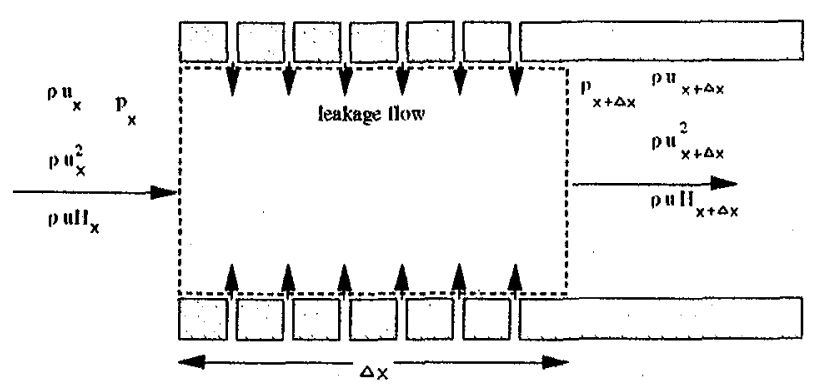

Figure 3.2.2 Leakage Flow Model 
It is not obvious from equations 3.2 .2 and 3.3.3 that the source term is a function of the conserved vector $w$. More importantly, the form of the Jacobian $[B]$ is difficult to obtain directly. It can however, be obtained through use of the chain rule, namely

$$
[B]=\frac{\partial \underline{S}}{\partial \underline{w}}=\frac{\partial \underline{S}}{\partial \underline{u}} \frac{\partial \underline{u}}{\partial \underline{w}}
$$

where $\underline{u}$ is the primitive variable vector $(p, \rho, u)^{T}$.

\section{$\underline{4.0 \text { Results }}$}

The results to be presented will compare predictions from the model described in the preceding sections with two laboratory wave rotor experiments. Both experiments utilize the so called divider cycle (Figure 4.1). In this cycle, the working gas (air in both cases) enters the wave rotor through one port and exits through two others. In one of the exit ports the stagnation pressure of the gas is higher than that which enters the wave rotor, in the other it is lower. Although the practical utility of such cycles is debatable, they serve as excellent vehicles for wave rotor studies because they are relatively simple. In particular, they do not require external heating devices such as a burner, which would be needed in a full wave rotor gas generator experiment. Furthermore, wave rotor divider cycles clearly illustrate the concept of work exchange through unsteady wave propagation.

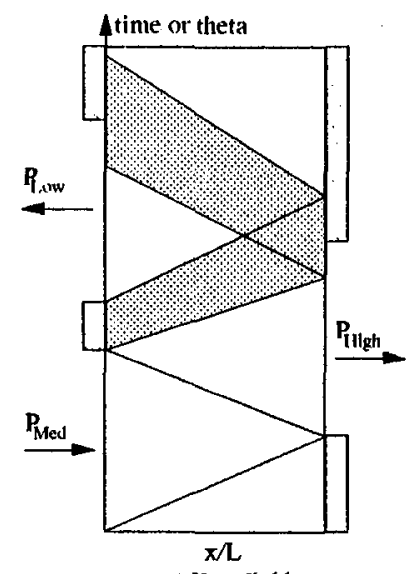

(a) Kentfield

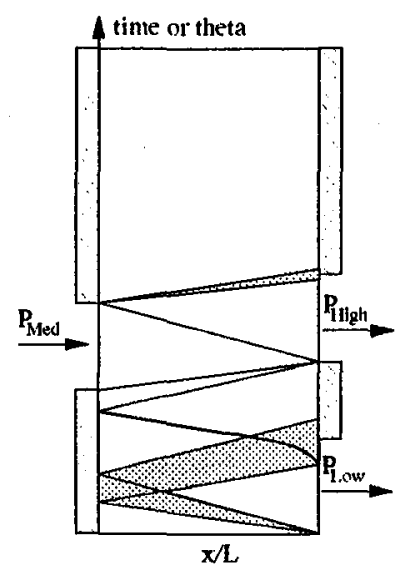

(b) NASA
Figure 4.I Proposed Experimental Wave Diagrams

The first experiment to be discussed is currently being conducted at NASA's Lewis Research Center. This wave rotor is highly instrumented making it possible to obtain both steady data in the ports and unsteady data from several $x / L$ positions on one of the tubes as it rotates through the cycle. The data which will be presented was obtained from a single operating point. Unfortunately, just after the experiment began operation, the supply air to the entire facility was turned off for repairs. The experiment will resume in several months. The second experiment was performed by J. A. C. Kentfield in the mid 1960 ' $\mathrm{s}^{(9)}$.
There is no unsteady data available from this experiment; however, the steady results are extensive and as such, can be used to test the capabilities of the model over a broad range of conditions. Figure 4.1 shows the simplified wave diagrams which the two experiments were intended to follow. Table 4.1 shows the relevant geometrical dimensions and the gas conditions used in the experiments. It should be noted that the two experinents represent relative extremes of the various effects that are being modelled. Kentfield's passages had large cross sections, were short, and had small end clearances. This made the frictional and leakage effects small and the finite opening time effects large. The NASA experiment on the other hand, has passages with very small cross sections and relatively large leakage paths.

\begin{tabular}{|l|l|l|}
\hline & Kentfield & \multicolumn{1}{|c|}{ NASA } \\
\hline Mean Tube Width, b & $0.60^{\prime \prime}$ & $0.25^{\prime \prime}$ \\
\hline Tube Height, $\mathrm{h}$ & $2.20^{\prime \prime}$ & $0.40^{\prime \prime}$ \\
\hline Rotor O.D., D & $8.00^{\prime \prime}$ & $12.00^{\prime \prime}$ \\
\hline Rotor Length, $\mathrm{L}$ & $11.00^{\prime \prime}$ & $18.00^{\prime \prime}$ \\
\hline End Clearance, $\delta$ & $0.007^{\prime \prime}$ & $0.01^{\prime \prime}$ ports \\
& & $0.02^{\prime \prime}$ walls \\
\hline Rotor Speed, $w$ & $6000 \mathrm{rpm}$ & $4150 \mathrm{rpm}$ \\
\hline Cycles/Revolution & 3 & 1 \\
\hline $\mathrm{r}_{\mathrm{OM}}$ & $555 \mathrm{R}$ & $637 \mathrm{R}$ \\
\hline $\mathrm{P}_{\mathrm{OM}}$ & & $22.41 \mathrm{psia}$ \\
\hline $\mathrm{P}_{\mathrm{OL}}$ & $14.70 \mathrm{psia}$ & \\
\hline$\alpha$ Equation 3.1.4 & 2.579 & 2.553 \\
\hline
\end{tabular}

Table 4.1 Experimental Parameters

The model was tested in the following manner. The geometric parameters were entered into the code along with the inlet stagnation conditions and exit static pressures. Also entered was a guess at the pressure and temperature in the cavity of the wave rotor. These values are used in the leakage calculations. The code, which actually follows a single tube, was then run through a cycle. As it computed, a running sum of the mass momentum and energy fluxes in the ports at each time step was kept as well as a sum of the total leakage mass and energy flow into the cavity. At the end of the cycle, the cavity pressure and temperature were updated using the leakage sums in a simple lumped parameter model of the cavity region, and the cycle was run again. This process was repeated until the cavity pressure and temperature reached a steady value, and the total mass flow into the wave rotor matched the total outflow. At this point the integrated port fluxes were used to calculate a representative uniform fluid state, averaged using a constant area mixing calculation ${ }^{(1)}$. This state was then used to obtain the outflow stagnation conditions. The 
condition of steady, matched flow in the ports is termed the limit cycle of the wave rotor for a specified set of port boundary conditions. It is the wave cycle which any tube on the rotor will continually repeat. In comparing the model with the Kentfield experiment a rather crude grid spacing of $\Delta x / L=0.02$ was used in order to reach the limit cycle condition as quickly as possible. The value of $\alpha$ in equation 3.1.4 was chosen so as to obtain the best overall match to the data. For comparison with the present NASA experiment, a finer grid spacing of $\Delta x / L=0.01$ was used and the value of $\alpha$ was chosen such that the computed ratio of high pressure exit mass flow to input mass flow, $\beta$ matched the experiment. The two values of $\alpha$ are listed for comparison in Table 4.1. It is seen that they are quite close, despite the very different geometries of the two experiments. This result lends some confidence to the analysis of the viscous effects.

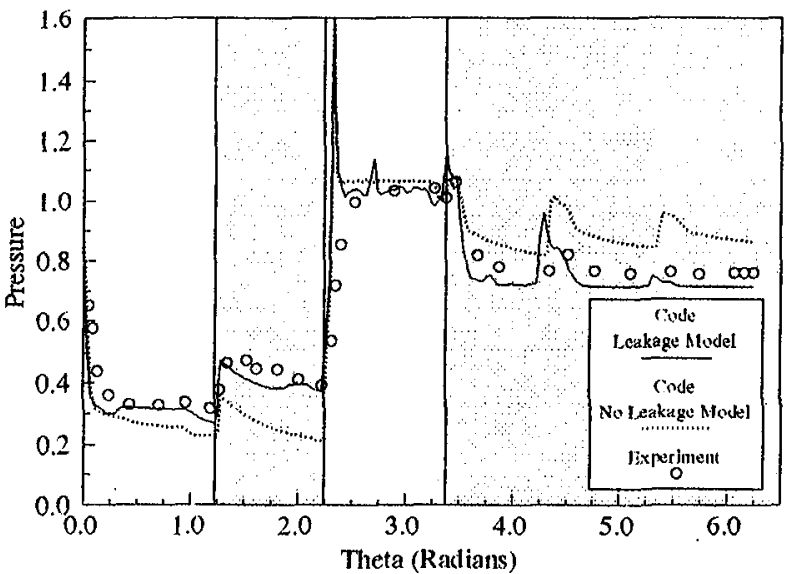

Figure 4.2 Unsteady Pressure Trace at $\mathrm{x} / \mathrm{L}=.975$

Tigure 4.2 shows a pressure trace measured in the tube of the NASA experiment, as it rotated through the cycle, at a location corresponding to $2.5 \%$ from the right side of Figure 4.1(b). All of the data has been nondimensionalized by the inlet stagnation pressure. The circular symbols represent the experimental data, the solid line was obtained using the model with friction and leakage effects. 'The shaded regions indicate those portions of the cycle where the end of the tube nearest the transducer was adjacent to a wall. It can be seen that there is excellent agreement between predicted and actual results. Furthermore, it was found that the predicted mass flow through the rotor was only $8.3 \%$ higher than the measured flow rate. This discrepancy might be accounted for by considering the blockage caused by the thickness of the tube walls. For reference, the dotted line in the figure represents the model output with only frictional effects. Although the timing of the waves appears correct the overall match with the experimental data is much worse. Furthermore, it was only possible to obtain the experimental mass flow split, $\beta$ if the friction coefficient $\alpha$ was increased 2.24 times over the case with leakage modelled. This result indicates that leakage flows have a considerable effect on the wave pattern.

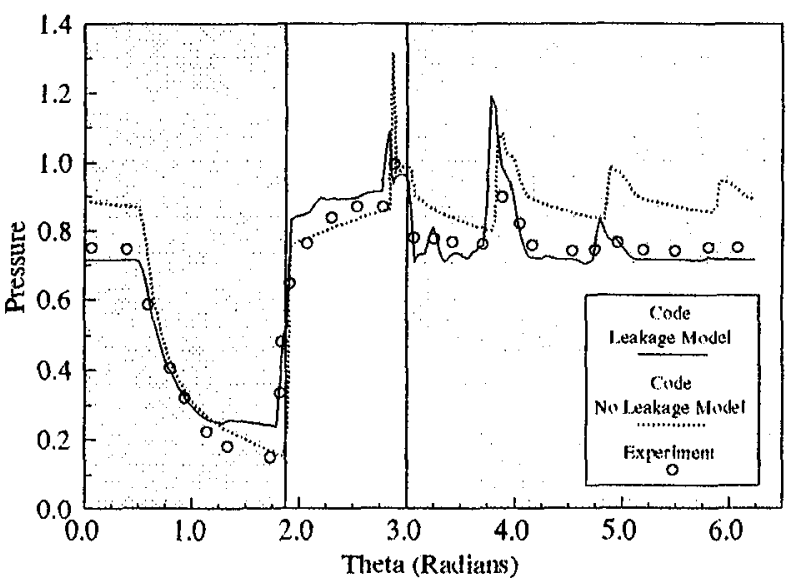

Figure 4.3 Unsteady Pressure Trace at $x / L=.250$

Figures 4.3 and 4.4 show the same comparisons between model and experiment for the $x / 2$ locations of .025 and .50 respectively, as measured from the left side of Figure 4.1(b). Again, the agreement is quite good. It is noted that the predicted pressure in the tube at the end of the cycle is slightly lower than the experimental pressure in all three figures. Also, the predicted pressure in Figure 4.3, just before the opening of the inlet port is too high. These discrepancies may be due to improperly specified clearances (values used in the model were obtained from specs, not measured) or errors resulting from the assumptions of leakage model itself.

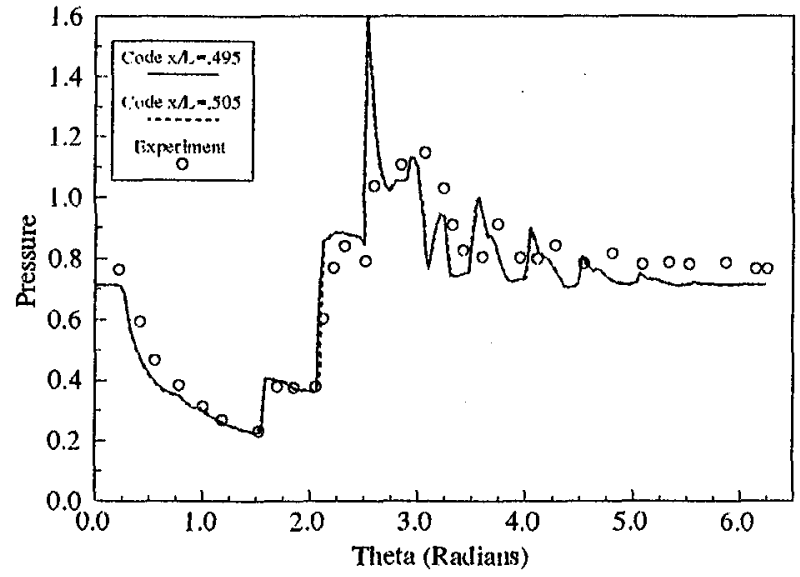

Figure 4.4 Unsteady Pressure 'Trace at $x / L=.500$

Figure 4.5 shows the results of Kentfield's experiment compared to the model predictions. Here, the ratio of the high pressure exit stagnation pressure to the inlet (medium pressure) stagnation pressure is plotted as a function of the ratio of the low pressure exit stagnation pressure to the inlet stagnation pressure for various values of the mass flow ratio $\beta$ (high pressure port mass flow to total mass flow). The agreement between predicted and experimental results is again, quite good. The largest discrepancies appear in the lower right portion of the plot. In this region, the mass flow, and corresponding port velocities are quite low. The circumferential component of velocity brought about by the rotation of the tubes is actually larger 
than the axial component generated by the waves and it is probable that this has a substantial effect on the performance. It is interesting to note that the lines of data all rise to the left and then abruptly stop. Kentfield pointed out that beyond this point the flow rate could not be increased. The same phenomenon was found in the model, although at slightly higher pressure.

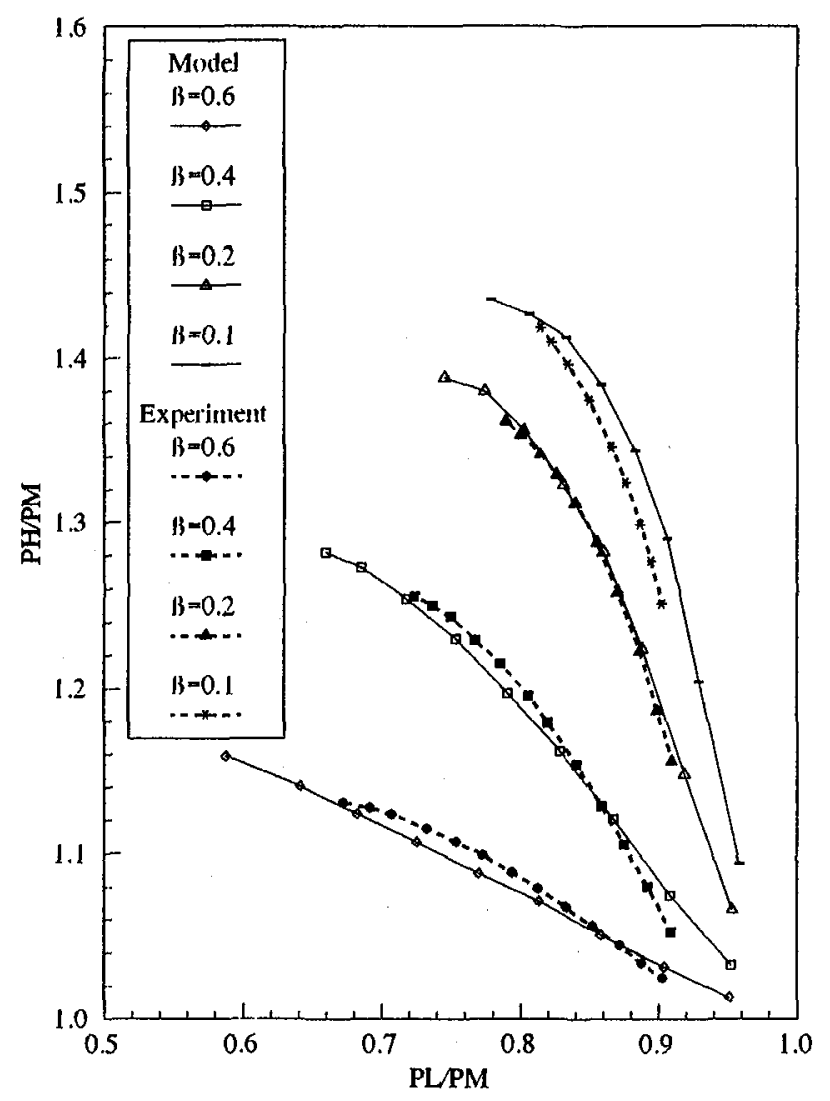

Figure 4.5 Kentfield's Divider Performance Curves

\subsection{CONCLUSIONS}

A numerical wave rotor model has been described which successfully predicted the behavior of two experiments with quite different geometries. The model is relatively simple in that it is one-dimensional, but sufficiently detailed to include the effects of viscosity, leakage and finite opening time. It requires relatively little computing time on a standard VAX mainframe (4.13 seconds CPU time for one cycle with 50 cells on a VAX 9410) and is therefore quite suitable for preliminary design purposes as well as analysis of existing wave rotors.

\section{REFERENCES}

1. Paxson, D. E., "A General Numerical Model for Wave Rotor Analysis", NASA TM 105740, 1992.

2. Eidelman, S., "The Problem of Gradual Opening in Wave Rotor Passages", Journal of Propulsion, 1985, Vol. 1, No. 1, pp 23-27.
3. Taussig, R. T., "Energy Exchanger Performance and Power Cycle Evaluation-Experiments and Analysis", Final Report DOE/ER/01084--T1, 1980.

4. Thompson, P. A., Compressible Fluid Dynamics, Department of Mechanical Engineering Rensselaer Polytechnic Institute, 1988.

5. Press, William, H., et al, Numerical Recipes, Cambridge University Press, 1986.

6. Lax, P. D., and Wendroff, B., "Difference Schemes for Hyperbolic Equations With High Order of Accuracy", Communications in Pure and Applied Mathematics, 1964, vol. 17, pp 381-398.

7. Roe, P. L., "Characteristic Based Schemes for the Euler Equations", Annual Review of Fluid Mechanics, 1986, vol. 18, pp 337-65.

8. Schlichting, Hermann, Boundary Layer Theory, $7^{\text {th }}$ Edition, McGraw-Hill Book Company, 1979.

9. Kentfield, J. A. C., "An Examination of the Performance of Pressure Exchanger Equalizers and Dividers", PhD Thesis, U. of London, 1963 (also in Journal of Basic Engineering, September, 1969). 
Public reporting burden for this collection of information is estimated to average 1 hour per response, including the time for reviewing instructions, searching existing data sources, gathering and maintaining the data needed, and completing and reviewing the collection of information. Send comments regarding this burden estimate collection of information, including suggestions for reducing this burden, to Washington Headquarters Services, Directorate for information Operations and Reports, 1215 Jefferson Davis Highway, Suite 1204, Arlington, VA 22202-4302, and to the Office of Management and Budget, Paperwork Reduction Project (0704-0188), Washington, DC 20503.

\begin{tabular}{|l|c|r|}
\hline 1. AGENCY USE ONLY (Leave blank) & $\begin{array}{c}\text { 2. REPORT DATE } \\
\text { October } 1992\end{array}$ & $\begin{array}{r}\text { 3. REPORT TYPE AND DATES COVERED } \\
\text { Technical Memorandum }\end{array}$ \\
\hline
\end{tabular}

4. TITLE AND SUBTITLE

An Improved Numerical Model for Wave Rotor Design and Analysis 5. FUNDING NUMBERS

6. AUTHOR(S)

WU-505-62-10

Daniel E. Paxson and Jack Wilson

8. PERFORMING ORGANIZATION REPORT NUMBER

National Aeronautics and Space Administration

Lewis Research Center

Cleveland, Ohio 44135-3191

E-7398

9. SPONSORING/MONITORING AGENCY NAMES(S) AND ADDRESS(ES)

10. SPONSORING/MONITORING AGENCY REPORT NUMBER

National Aeronautics and Space Administration

Washington, D.C. 20546-0001

NASA TM-105915

AIAA-93-0482

11. SUPPLEMENTARY NOTES

Prepared for the 31 st Aerospace Sciences Meeting sponsored by the American Institute of Aeronautics and Astronautics, Reno, Nevada, January 11-14, 1993. Daniel E. Paxson, NASA Lewis Research Center, Cleveland, Ohio, and Jack Wilson, Sverdrup Technology, Inc., 2001 Aerospace Parkway, Brook Park, Ohio 44142. Responsible person, Daniel E. Paxson, (216) 433-8334.

12a. DISTRIBUTION/AVAILABILITY STATEMENT

12b. DISTRIBUTION CODE

Unclassified - Unlimited

Subject Category 07

13. ABSTRACT (Maximum 200 words)

A numerical model has been developed which can predict both the unsteady flows within a wave rotor and the steady averaged flows in the ports. The model is based on the assumptions of one-dimensional, unsteady, perfect gas flow. Besides the dominant wave behavior, it is also capable of predicting the effects of finite tube opening time, leakage from the tube ends, and viscosity. The relative simplicity of the model makes it useful for design and optimization, as well as analysis, of wave rotor cycles for any application. This paper will discuss some details of the model and will then present comparisons between the model and two laboratory wave rotor experiments.

14. SUBJECT TERMS
Wave rotor; Computational fluid mechanics

17. SECURITY CLASSIFICATION OF REPORT

Unclassified
18. SECURITY CLASSIFICATION OF THIS PAGE

Unclassified
19. SECURITY CLASSIFICATION OF ABSTRACT Unclassified 
National Aeronautics and Space Administration

Lewis Research Center Cleveland, Ohio 44135

Official Business

Penalty for Private Use $\$ 300$

\section{ADDRESS CORRECTION REQUESTED}

|||||

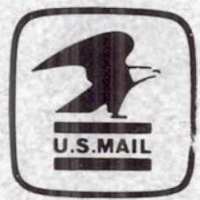

Postage and Fees Paid National Aeronautics an

Space Administration NASA 451 